\title{
Feasibility of Lateral Flow Assays for Urinary Biomarkers to Nicotine Delivery Products
}

\author{
Michael P. Nguyen ${ }^{1}$, Jonathan Gary ${ }^{1}$, Jack Sonoda ${ }^{1}$, Mitchell Low ${ }^{1}$, Christian Ring ${ }^{1}$, \\ and Jeffrey J. Bishop ${ }^{1}$
}

${ }^{1}$ Lumos Diagnostics, Carlsbad, CA, USA

E-mail: mike.nguyen@lumosdiagnostics.com

Submitted October 4, 2021

\section{Graphical Abstract}

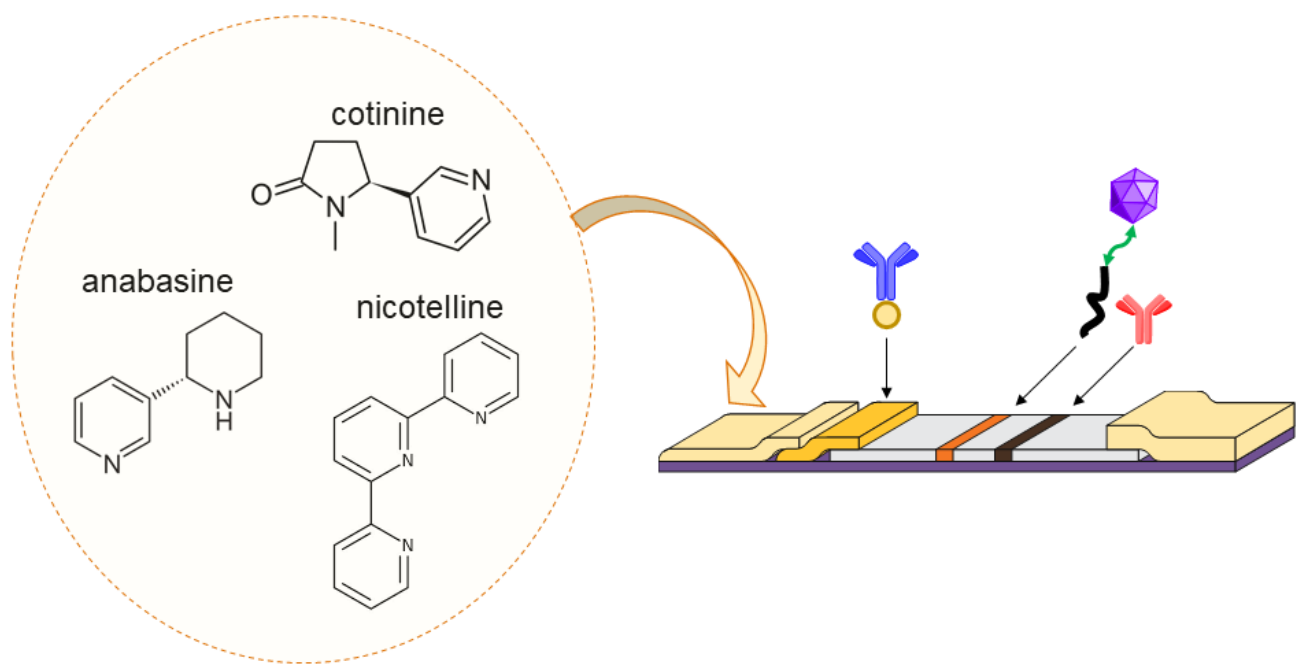

\section{Abstract}

The goal of this work was to develop lateral flow immunoassays (LFAs) to biomarkers related to nicotine-containing products such as combusted tobacco, oral tobacco, and e-cigarettes. LFAs were screened to determine how well they discriminate users of nicotine-containing products from non-users. Additional analyses assessed if any developed LFAs show signs of distinction between the various use-groups (combusted tobacco, oral tobacco, and e-cigarettes). The following describes the development of cotinine, anabasine, and nicotelline LFAs as well as the test and analyses from 212 individual urine specimens.

Keywords: lateral flow assay, nicotine, tobacco, metabolites, urine

\section{Introduction}

Smoking is the single most preventable cause of death and disability in the world today. ${ }^{1}$ More than 16 million
Americans currently live with diseases caused by smoking, and it is predicted that there will be 5.6 million premature deaths of Americans under the age of 18 if smoking continues at the current rate. ${ }^{1}$ This issue is compounded by new nicotine- 
Table 1. Materials list per LFA with their respective dimensions per strip. Components pretreated. ${ }^{*}$

\begin{tabular}{|c|c|c|c|}
\hline Material & Cotinine & Anabasine & Nicotelline \\
\hline Detector Reagent & Recombinant Antibody & $\mathrm{rFab}$ & rFab \\
\hline Particle & Gold Nanoshell & Gold Nanoshell & Colloidal Gold \\
\hline Backing Card & \multicolumn{3}{|c|}{ Lohmann LC-58778 (73.5 mm) } \\
\hline Membrane & Unisart CN95 (25 mm) & Cytiva FF120 (25 mm) & Cytiva FF120 (25 mm) \\
\hline Waste Pad & \multicolumn{3}{|c|}{ Ahlstrom $440(26 \mathrm{~mm})$} \\
\hline Conjugate $\mathrm{Pad}^{\star}$ & \multicolumn{3}{|c|}{ Ahlstrom $8980(10 \mathrm{~mm})$} \\
\hline Sample Pad ${ }^{*}$ & \multicolumn{3}{|c|}{ Ahlstrom 1668HV+ (21 mm) } \\
\hline
\end{tabular}

containing products such as e-cigarettes that have infiltrated youth culture. ${ }^{2}$ On top of wide-spread use, dishonesty in selfreported nicotine use is common making cessation studies difficult to conduct. ${ }^{3-6}$ Gold-standard analyses to objectively report use exist to aid these cessation studies (eg. HPLCtandem mass spectrometry), however, they are expensive $(\geq \$ 47$ per test) and immobile, making them not suitable for high-throughput testing. ${ }^{7}$ Lateral flow immunoassays (LFAs) provide a rapid, simple, portable, and inexpensive method to measure specific analytes in various biological matrixes. ${ }^{8}$ The specificity comes from employing antibodies which are developed to detect a biomarker of interest. Therefore, LFAs are a good candidate for swiftly detecting these biomarkers on a scale relevant to a larger cessation study. Commercially available cotinine LFAs for urine exist and cost approximately $\$ 2$ per test. Cotinine is the primary metabolite of nicotine in urine, making it a reliable marker to inform about use of any nicotine-containing product. ${ }^{7}$ While cotinine is a proven biomarker for indicating nicotine use versus nonuse, it cannot distinguish use between other nicotine-containing products such as combusted tobacco, oral tobacco, or e-cigarettes. If a panel of LFAs could be developed that discriminate between use groups, cessations studies could then monitor how participants are switching between tobacco versus nontobacco-containing product use (i.e. combusted and oral tobacco versus e-cigarette and nicotine replacements). Literature supports the feasibility of additional biomarkers beyond cotinine that may also inform use of various nicotinecontaining products; $;{ }^{79-11}$ therefore we set out to investigate using a LFA platform to detect these biomarkers that could identify nicotine-product use.

\section{Experimental section}

2.1 Chemical synthesis. For the anabasine and nicotelline assays, KLH-antigen reagents for immunization and BSAantigen reagents for clone selection and LFA development were synthesized by CellMosaic (Woburn, MA).
2.2 rFab generation. De novo $\mathrm{rFabs}$ were synthesized at Antibody Design Labs (San Diego, CA). The rFabs were Cterminally Avi-tagged.

2.3 Particle conjugation. Two types of nanoparticle labels were used in this study. The first was a $150 \mathrm{~nm}$ diameter carboxyl-coated gold nanoshell (GNS) (GSXR150) from NanoComposix (San Diego, CA) and the second was an 80 $\mathrm{nm}$ diameter streptavidin-coated colloidal gold (CG) (CGSTV-0600) from Arista Biologicals Inc (Allentown, PA). Antibody-particle conjugations for the GNS labels were completed by following NanoComposix's "BioReady ${ }^{\mathrm{TM}} 150$ $\mathrm{nm}$ Carboxyl Gold Nanoshells Covalent Conjugation Protocol" (Version 1.4). For the CG conjugations, the biotinylated $\mathrm{rFabs}$ were first brought to a concentration of 1 $\mathrm{mg} / \mathrm{mL}$ with a $1 \mathrm{x}$ PBS solution then added to the CG at OD1. After mixing, a 20x molar excess of biotin was added to the mixture and vortexed. The quenched CG particles were then centrifuged and resuspended in a blocking buffer until use.

2.4 Specimen collection. A prospective collection of 212 individual urine specimens from users and non-nicotine users was completed between July 2020 and April 2021 by Discovery Life Sciences (Los Osos, CA). All enrollees selfidentified as non-users (112 samples) or single-product users (101 samples). Within the group of users, the highest number of samples came from the combusted tobacco (71) use-group with the remaining specimens coming from the following usegroups: e-cigarette (17) and oral tobacco (12).

2.5 Strip materials and preparation. For each of the LFAs, a Lohmann backing card was used to support the Ahlstrom 1668HV+ sample pad, Ahlstrom 8980 conjugate pad, and Ahlstrom 440 waste pad. The cotinine LFA specifically used a Unisart CN95 membrane, a GNS label, and a recombinant detector antibody. The anabasine LFA used a Cytiva FF120 membrane, a GNS label, and a rFab detector. The nicotelline LFA used a Cytiva FF120 membrane, CG label, and a rFab detector. Table 1 lists the materials used for each developed LFA. 
Figure 1. Process for vetting biomarker selection and outcomes.

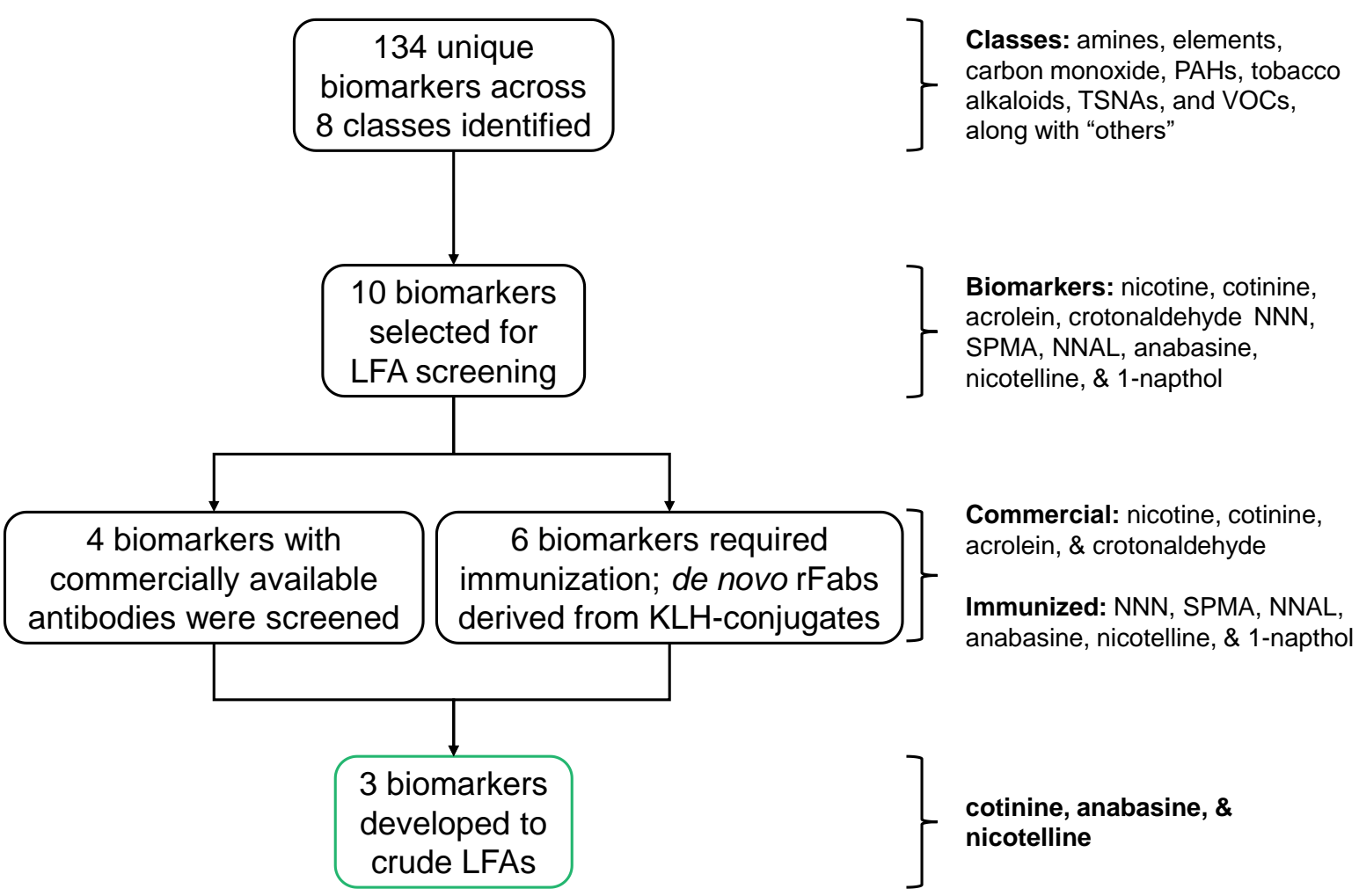

The strip materials requiring pre-treatment were the conjugate pads and sample pads. The conjugate pad treatment buffer was composed of blocking components and surfactants to ensure uniform and complete release of the conjugate. A $100 \mathrm{mM}$ phosphate solution buffered to $\mathrm{pH} 8.0$ served as a sample pad buffer to ensure the urine reached a standard $\mathrm{pH}$ as it interacted with the nanoparticle conjugate and test line reagents. After the respective pads were treated and allowed to dry, the pads were stored in a desiccated environment at room temperature until use.

A BioDot XYZ3050 was used to dispense the test lines and control lines for all LFAs. The test and control line reagents were diluted using a 1x PBS solution and dispensed at a rate of $0.5 \mu \mathrm{L} / \mathrm{cm}$. The treated conjugate pad was laminated to the backing card with a $2 \mathrm{~mm}$ overlap onto the membrane and the sample pad was laminated onto the backing card to maintain a $3.5 \mathrm{~mm}$ overlap onto the conjugate pad. Once the full card was assembled, a guillotine-style cutter prepared $4 \mathrm{~mm}$ wide test strips. Upon final assembly, the test strips were placed in a desiccated environment at room temperature until use.

2.6 Standard curve generation. A standard curve was prepared for each of the three LFAs, cotinine, anabasine, and nicotelline. These curves were generated by spiking the analyte into a pool of negative matrix (urine). The concentrations for the cotinine standard curve were the following: $0,100,250,500,1000,2500,5000 \mathrm{ng} / \mathrm{mL}$. The concentrations for the anabasine standard curve were the following: $0,15,30,45,60,100,300 \mathrm{ng} / \mathrm{mL}$. The concentrations for the nicotelline standard curve were the following: $0,10,50,100,500,2000 \mathrm{ng} / \mathrm{mL}$. Each of the standard curves were fit to a four parameter logistic (4PL) regression using JMP statistical software (JMP Trial 16.0.0). The limit of detection (LOD) for each of the cotinine, anabasine, and nicotelline LFAs were calculated to be 417 $\mathrm{ng} / \mathrm{mL}, 10 \mathrm{ng} / \mathrm{mL}$, and $26 \mathrm{ng} / \mathrm{mL} .^{12}$

2.7 Patient specimen testing scheme. Each of the 212 patient specimens were tested in triplicate over three consecutive days on each LFA. Each LFA was optimized for 60 to $100 \mu \mathrm{L}$ of sample volume and was read after $15 \mathrm{~min}$ on a Leelu benchtop test strip reader (Lumos Diagnostics, Carlsbad, CA) to optically scan and provide a quantitative measurement of the test line intensity. Prior to testing individual patient samples, a standard curve prepared in negative urine was run to confirm suitable day-to-day functionality and ensure that no degradation of the strip quality occurred since the testing was completed over multiple days.

To eliminate any possible testing bias, all test specimens were deidentified and randomized so that the LFA operators were blind to expected outcomes. To further reduce bias, the second day of testing was completed in reverse random order. 
Figure 2. Dose-response plots of cotinine, anabasine, and nicotelline LFAs in urine with each of the three replicates plotted per concentration. Inset plots show detail at lower concentrations. Reported concentrations for cotinine, anabasine, and nicotelline* for non-users and nicotine-product users are also found below. $7,13,19$ *Denotes values are measured as $\mathrm{N}$-oxides.

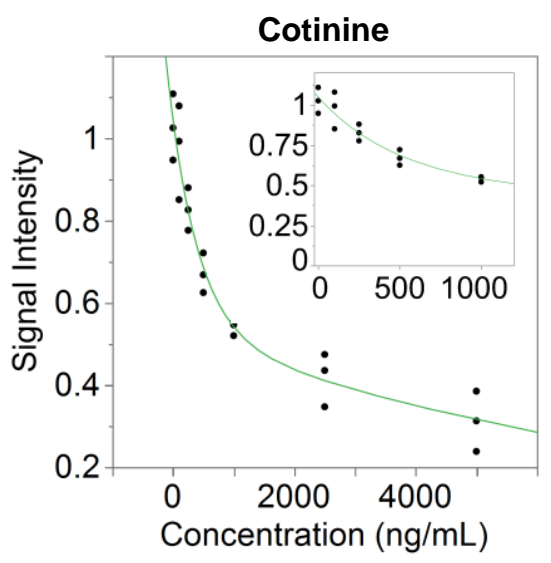

Nonuser: $<50 \mathrm{ng} / \mathrm{mL}$ User: $1000-8000 \mathrm{ng} / \mathrm{mL}$ LOD: $417 \mathrm{ng} / \mathrm{mL}$

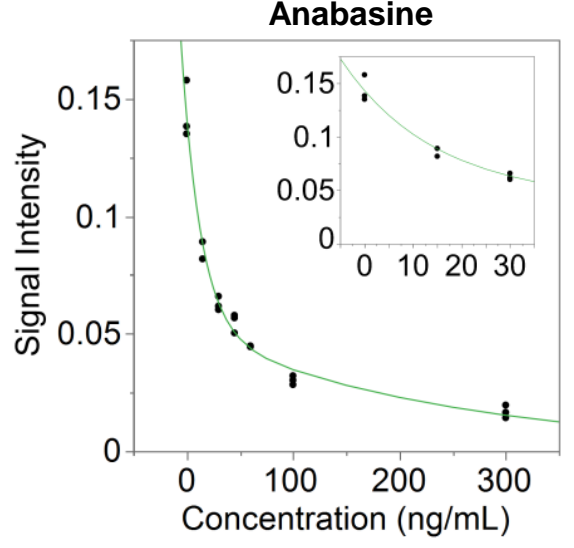

Nonuser: $<2 \mathrm{ng} / \mathrm{mL}$ User: $10-500 \mathrm{ng} / \mathrm{mL}$ LOD: $10 \mathrm{ng} / \mathrm{mL}$

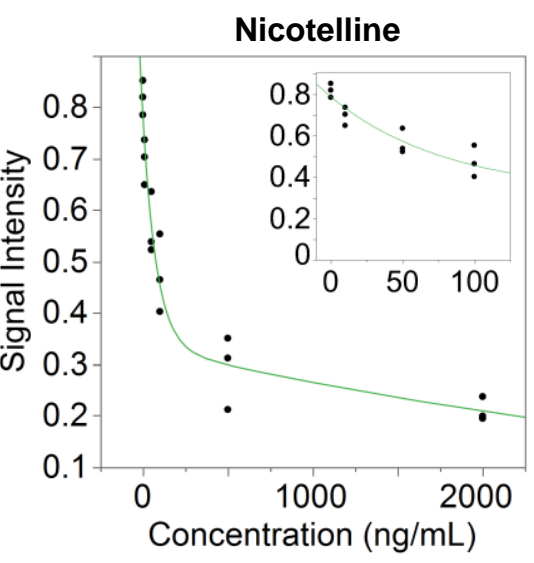

Nonuser*: $<0.1 \mathrm{ng} / \mathrm{mL}$ User*: $0.1-0.15 \mathrm{ng} / \mathrm{mL}$ LOD: $26 \mathrm{ng} / \mathrm{mL}$
2.8 Data analysis method. All patient sample data was first measured as signal intensity measured by the Leelu benchtop test strip reader. The signal for each sample was then compared to the day's calibration curve and a concentration was extrapolated. Samples that were extrapolated as negative concentrations (i.e. the signal was higher than that of the signal from the blank calibrator) were set to $0 \mathrm{ng} / \mathrm{mL}$. Samples that were extrapolated as concentrations beyond the calibration curve (i.e. signals were lower than that of the signal from the highest concentration calibrator) were truncated to the highest calibrator concentration; these concentrations for the truncated samples were $5000 \mathrm{ng} / \mathrm{mL}, 300 \mathrm{ng} / \mathrm{mL}$, and 2000 $\mathrm{ng} / \mathrm{mL}$ for the cotinine, anabasine, and nicotelline LFAs, respectively. The mean concentration for the three days was calculated. This data was used to create a two-tailed Student's $\mathrm{t}$-test (t-test) comparing the significance between use-groups. For each LFA, this analysis was completed using JMP.

In addition to the t-tests, receiver operating characteristic (ROC) curves were calculated using JMP to further assess LFA performance. Key performance metrics gathered from these ROC curves highlight the LFA's area under the curve (AUC), as well as clinical sensitivity (sensitivity) and clinical specificity (specificity) at the optimal cutoff (i.e. maximum sum of sensitivity and specificity).

\section{Results}

3.1 Biomarker selection. An extensive literature search to assess which biomarkers would provide the highest likelihood for classifying nicotine-product use was completed. A key finding from this effort was that no one biomarker has yet been identified that is capable of discrminating all use-groups, therefore, our aim was to target a panel of multiple biomarkers for assay development. A total of 134 unique biomarkers across eight compound classes (amines, elements, carbon monoxide, polyaromatic hydrocarbons (PAHs), tobacco alkaloids, tobacco-specific nitrosamines (TSNAs), and volatile organic compounds (VOCs), along with "others" for those that did not fit into the other groups) were initially identified.

Many of these biomarkers can be found in multiple biological matrixes, however, $74 \%$ of the studies reported use of urine. In addition, urine collections are non-invasive making these samples easier to obtain. Therefore, urine was chosen as the targeted matrix.

Based on several criteria, including suitability for LFA development, variety of compound type, and availability of reagents, the following 10 biomarkers were selected for LFA generation: nicotine, cotinine, acrolein, crotonaldehyde, Nnitrosonornicotine (NNN), S-phenylmercapturic acid (SPMA), 4-(methylnitrosamino)-1-(3-pyridyl)-1-butanol (NNAL), anabasine, nicotelline, and 1-napthol. For the NNN, S-PMA, NNAL, anabasine, nicotelline, and 1-naphthol biomarkers, there were no predicate reagents. Therefore, these biomarkers required the generation of of novel reagents (i.e. KLH-coupled immunogens, BSA-coupled test line material, as well as rFabs; see section 2.1 and 2.2). Figure 1 shows the vetting process for biomarkers to screen on a LFA format and the outcomes.

3.2 Reagent screening. LFAs for 10 biomarkers (nicotine, cotinine, acrolein, crotonaldehyde, NNAL, S-PMA, 
anabasine, NNN, 1-napthol, \& nicotelline) were screened using commercially available and/or de novo reagents. Where available, multiple antibodies or rFabs were explored for each biomarker on LFAs. Providing a dose-response in spiked urine near endogenous levels was the key performance metric to select final antibodies and rFabs. After this screening was completed for 28 antibodies and rFabs across the 10 biomarkers, the cotinine, anabasine, and nicotelline LFAs emerged to satisfy the required performance. For the cotinine LFA, a commercially available recombinant antibody and BSA-antigen were used. Both of the anabasine and nicotelline LFAs were developed with de novo rFabs and BSA-antigen reagents.

After LFA optimization, a dose-response curve was generated for each of the LFAs using triplicate measurements in a negative urine matrix (Figure 2). The mean CVs for the cotinine, anabasine, and nicotelline dose-response curves were $5.5 \%, 6.8 \%$, and $12 \%$, respectively.

\subsection{Single assay significance from patient samples. The} 212 patient samples were tested on the three LFAs in triplicate. The data was organized by use-group and a t-test was performed. For each of the use-groups, the diamonds displayed in green represent the $95 \%$ confidence interval around the mean signal intensity. The cotinine LFA showed significance between nonusers and each of the individual user groups (combusted tobacco, e-cigarette, and oral tobacco) with p-values $\leq 0.05$ (Figure 3 ).

Figure 3. T-test for the cotinine LFA. For each of the use-groups, the diamonds displayed in green represent the $95 \%$ confidence interval around the mean signal intensity.

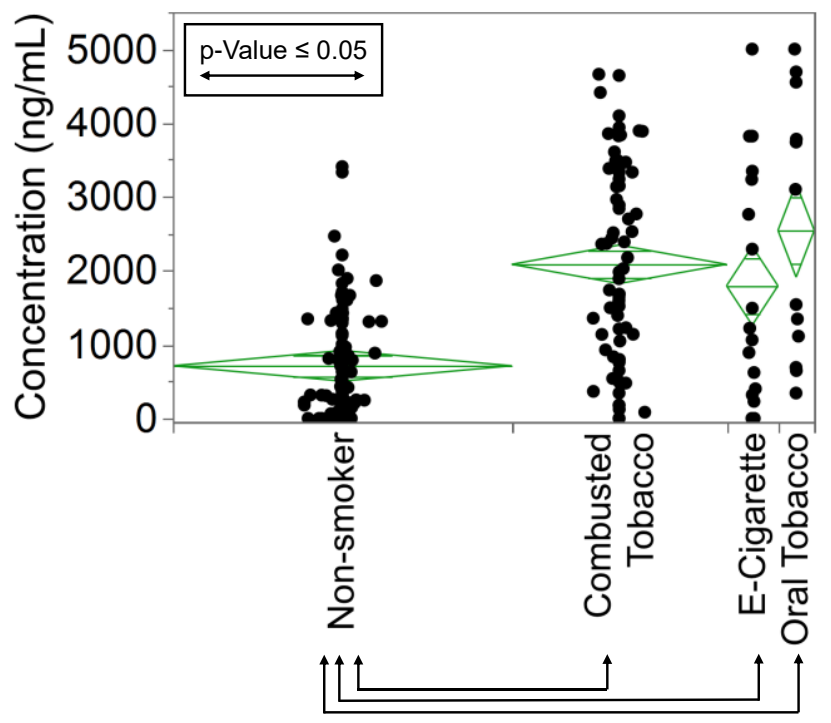

The t-test results for the anabasine LFA in Figure 4 shows the significance between urine from nonusers and
Figure 4. T-test for the anabasine LFA. For each of the use-groups, the diamonds displayed in green represent the $95 \%$ confidence interval around the mean signal intensity.

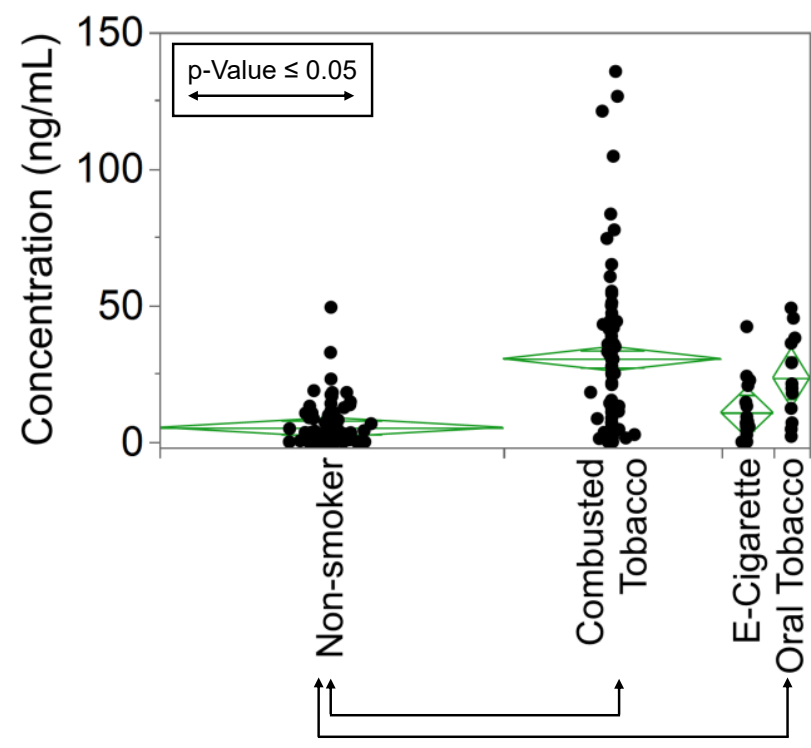

Figure 5. T-test for the nicotelline LFA. For each of the use-groups, the diamonds displayed in green represent the $95 \%$ confidence interval around the mean signal intensity.

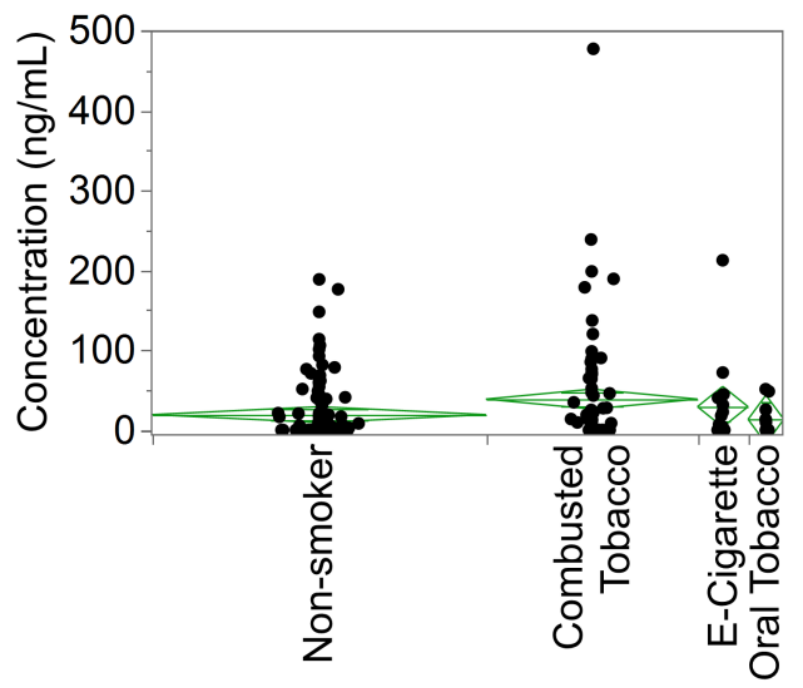

combusted tobacco users as well as nonusers from oral tobacco users based on a $95 \%$ confidence interval ( $p$-values $\leq 0.05$ ). The t-test comparing the results of the different usegroups on the nicotelline LFA is shown in Figure 5 and suggests no significance between any of the use-groups. 
Figure 6. ROC curve plots for the cotinine, anabasine, and nicotelline LFAs as well as a table for respective performance metrics. The yellow line makes contact at the cut-off value at a $45^{\circ}$ angle.
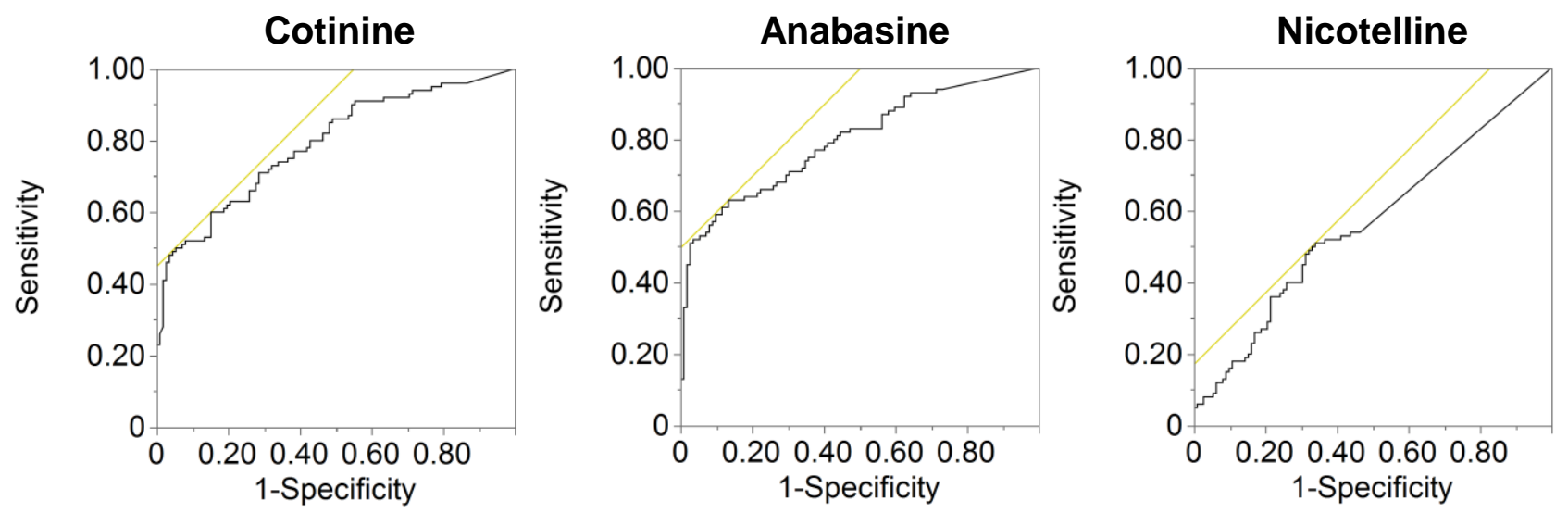

\begin{tabular}{|c|c|c|c|c|}
\hline \multicolumn{5}{|c|}{ Individual LFA Cut-off Values and Corresponding Performance Metrics } \\
\hline Assay & AUC & Cut-off (ng/mL) & Clinical Sensitivity & Clinical Specificity \\
\hline Cotinine & 0.78 & 1492 & 0.60 & 0.85 \\
Anabasine & 0.80 & 10.9 & 0.63 & 0.87 \\
Nicotelline & 0.56 & 8.6 & 0.51 & 0.66 \\
\hline
\end{tabular}

\subsection{Receiver operating characteristic (ROC) curve} analyses. Receiver operating characteristic (ROC) curves were calculated and their associated performance metrics were used to inform each LFA's ability to predict use of a nicotinecontaining product vs nonuse (Figure 6). For the cotinine, anabasine, and nicotelline LFAs, the AUC values were the following: $0.78,0.80$, and 0.56 , respectively. The sensitivity and specificity values for the cotinine and anabasine LFAs are similar for each, however, every metric is higher for the anabasine assay.

The cotinine and anabasine LFA data were combined and a ROC curve analysis was completed (Figure 7). The ROC curve from the combined data marginally improved the AUC value, sensitivity, and specificity to $0.84,0.70$, and 0.92 , respectively.

\section{Discussion}

As smoking continues to prevail as the leader in preventable deaths, public health agencies will continue to conduct cessation studies. LFAs that can distinguish nicotineproduct use versus nonuse could provide a convenient platform to aid in monitoring outcomes of these studies without relying on participant veracity. While a cotinine LFA can differentiate nonuse vs use of a nicotine-containing product, additional LFAs beyond cotinine that are capable of distinguishing between use-groups could also aid these studies in monitoring the use of tobacco-free alternative products (i.e. e-cigarettes and nicotine replacements over combusted tobacco and oral tobacco ones).

Therefore, we identified ten biomarkers and pursued assay development for them. From the ten, we developed three competitive LFAs to detect cotinine, anabasine, and nicotelline in urine. Cotinine is a metabolite of nicotine, and the other two are parent compounds found in tobacco. These LFAs were used to test 212 urine samples from four usegroups including combusted tobacco, oral tobacco, ecigarettes, and self-reported non-users of any of these products. The testing was done to assess the feasibility of distinguishing, broadly, users from non-users and perhaps possibly differentiating, more specifically, between usegroups.

Nicotelline has been proposed as a biomaker to discriminate combusted tobacco users from oral tobacco users. ${ }^{13,14}$ We proposed that a nicotelline LFA that could discriminate between combusted and oral tobacco users could aid results of the cotinine and anabasine LFA results. The ttest revealed that the nicotelline LFA showed no ability discern any of the use-groups, individually. The nicotelline LFA's LOD being just outside the clinical range may be attributed to the lack of significance. 
Figure 7. ROC curve plot for the combined LFA data as well as a table for respective performance metrics. The yellow line makes contact at the cutoff value at a $45^{\circ}$ angle.

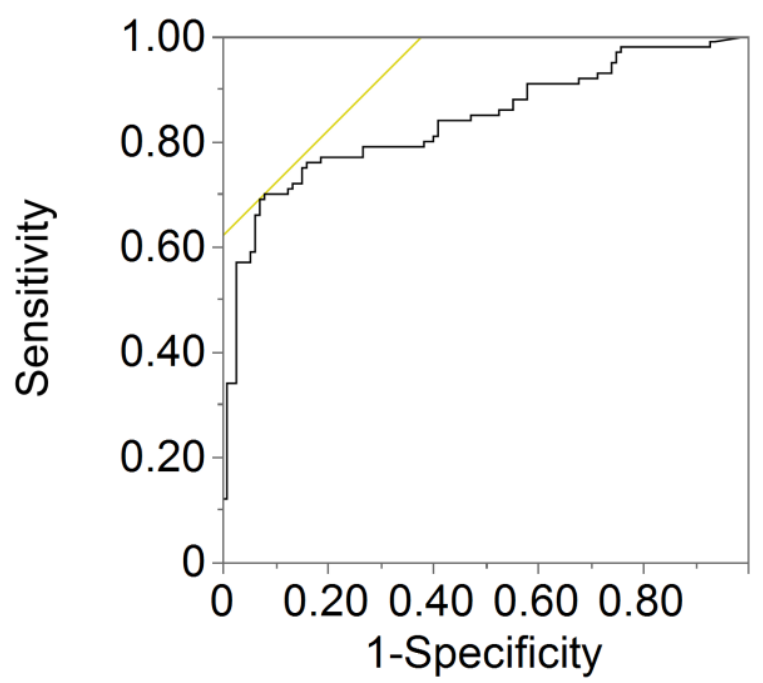

\begin{tabular}{|c|c|c|c|}
\hline \multicolumn{4}{|c|}{ Combined LFA Performance Metrics } \\
\hline Assay & AUC & $\begin{array}{c}\text { Clinical } \\
\text { Sensitivity }\end{array}$ & $\begin{array}{c}\text { Clinical } \\
\text { Specificity }\end{array}$ \\
\hline $\begin{array}{c}\text { Cotinine \& } \\
\text { Anabasine }\end{array}$ & 0.84 & 0.70 & 0.92 \\
\hline
\end{tabular}

In agreement with literature results, the cotinine LFA data replicated the ability to distinguish nonusers from users. $^{5,15-18}$ The data in Figure 3 shows the significance between nonusers and each of the user groups as cotinine is the main urinary metabolite for nicotine which is found in all of the products.

The anabasine LFA could also distinguish nonusers from combusted tobacco users and nonusers from oral tobacco users. No further distinctions could be made from nonusers to e-cigarette users; perhaps this is because anabasine is a tobacco-derived alkaloid and is only expected to be present in samples where a tobacco plant is part of the product. ${ }^{7,19}$

We then used ROC curve analysis to assess how anabasine compares with cotinine as a biomarker for nicotineproduct use. The anabasine LFA established a marginal improvement over the cotinine LFA in overall ability to distinguish users from nonusers demonstrated by the higher AUC, sensitivity, and specificity. Our next analysis was to determine if performance metrics would improve by combining the data from the two high performing LFAs, cotinine and anabasine. The combination of the two assays showed a small increase in the ability to distinguish users from non-users over the individual results. Another combined ROC curve was prepared using all three LFAs, cotinine, anabasine, and nicotelline; the results were not significantly different from those of the combined cotinine and anabasine LFA (data not shown).

4.1 Limitations \& future work. There were several limitations we encountered in this work regarding the assays and samples. After our feasibility screening of the ten LFAs we developed only three functional LFAs which made for a low likelihood of being able to definitively distinguish usegroups. Additionally, these LFAs were not fully optimized for the samples as demonstrated by a significant number of nonuser samples having stronger signals (i.e. stronger test line binding) than our signals for our blank samples in our calibration curves. Future work would consist of pursuing more LFAs by immunizing to more biomarkers as well as optimizing the LFAs to a larger subset of non-user urine samples to account for individual variation.

A limitation in the specimen collection was generating insufficient numbers of specimens for the ecigarette and oral tobacco use-groups. Key information unknown from the collection was the time of collection/urination number, frequency of use, time of last use, and second-hand exposure. Much of these collection issues derived from its conduction during the 2020 COVID19 pandemic. Further, there were no methods used to quantitate biomarker concentrations in individual samples therefore it was never confirmed if signal trends were solely attributed to the intended analyte. A follow-up collection would focus on the use-groups from which a minority of samples were first collected and ensure that all samples were paired with desired information.

While none of the LFAs displayed a statistical significance between any of the use-groups (e.g. combusted tobacco versus oral tobacco, etc.), there could be some distinction implications using multiple assays. Following the proposed work, it is possible a decision tree-type analysis could be employed.

4.2 Summary. This work set out to develop LFAs to discriminate users of nicotine-containing products from nonusers. After an extensive literature search, ten biomarkers were chosen to pursue LFA development toward; three of those biomarkers, cotinine, anabasine, and nicotelline were successful in generating competitive LFAs in a clinical urine matrix.

212 urine specimens from combusted tobacco, oral tobacco, e-cigarette, and nonusers were tested on each of the three LFAs. ROC curve analysis was completed for the cotinine and anabasine LFA data, individually, demonstrating good and similar clinical performance in distinguishing use from nonuse. The analysis was replicated on data combined from both the commercial cotinine and de novo anabasine 
LFAs, the outcomes however, only slightly improved the clinical performance.

A rapid, sensitive, and economical tool to detect and differentiate nicotine-product use may be valuable for objectively determining compliance with and the success of various cessation efforts and protocols. While this work has limitations, it serves as a proof of concept that additional work, with more analytes and improved analytical performance may still achieve nicotine use-group discrimination.

\section{Abbreviations}

LFA, lateral flow immunoassay; HPLC, highperformance liquid chromatography; GNS, gold nanoshell; CG, colloidal gold; PBS; phosphate buffered saline; 4PL, four parameter logistic; LOD, limit of detection; CV, coefficient of variance; $\mathrm{rFab}$, recombinant antigen-binding fragment; $\mathrm{PAH}$, polyaromatic hydrocarbon; TSNA, tobacco-specific nitrosamine; VOC, volatile organic carbon; NNN, Nnitrosonornicotine; S-PMA, S-phenylmercapturic acid; NNAL, 4-(methylnitrosamino)-1-(3-pyridyl)-1-butanol; KLH, keyhole limpet hemocyanin; BSA, bovine serum albumin; ROC, receiver operating characteristic; AUC, area under the curve.

\section{Acknowledgements}

This study was funded with a grant from the Foundation for a Smoke-Free World, Inc. ("FSFW"), a US nonprofit 501(c)(3) private foundation. For more information about FSFW, please visit its website. The authors would also like to thank Harry Scott, Norma Mendoza, Ashley Remigio, Joline Luo, Christina Monji, Phillip Bruce, and Sacha Dopheide for their contributions to this work.

\section{Conflict of interests}

The contents, selection, and presentation of facts, as well as any opinions expressed herein are the sole responsibility of the authors and under no circumstances shall be regarded as reflecting the positions of the Foundation for a Smoke-Free World, Inc. The authors have no conflicts to disclose.

\section{References}

(1) United States Department of Health and Human Services. The Health Consequences of Smoking - 50 Years of Progress A Report of the Surgeon General. A Rep. Surg. Gen. 2014, 1081.

(2) Services, U. S. D. of H. and H. E-Cigarette Use Among Youth and Young Adults. A Report of the Surgeon General. A Rep. Surg. Gen. 2016, 295.

(3) Gorber, S. C.; Schofield-Hurwitz, S.; Hardt, J.; Levasseur, G.; Tremblay, M. The Accuracy of SelfReported Smoking: A Systematic Review of the
Relationship between Self-Reported and CotinineAssessed Smoking Status. Nicotine Tob. Res. 2009, 11 (1), 12-24. https://doi.org/10.1093/ntr/ntn010.

Hilberink, S. R.; Jacobs, J. E.; van Opstal, S.; van der Weijden, T.; Keegstra, J.; Kempers, P. L. J.; Muris, J. W. M.; Grol, R. P. T. M.; de Vries, H. Validation of Smoking Cessation Self-Reported by Patients with Chronic Obstructive Pulmonary Disease. Int. J. Gen. Med. 2011, 4, 85-90. https://doi.org/10.2147/IJGM.S15231.

Yeh, E.; Levasseur, G.; Kaiserman, M. J. Evaluation of Urinary Cotinine Immunoassay Test Strips Used to Assess Smoking Status. Nicotine Tob. Res. Off. J. Soc. Res. Nicotine Tob. 2011, 13 (11), 1045-1051. https://doi.org/10.1093/ntr/ntr127.

Heo, H. C.; Byun, Y. S.; Sohn, S. H.; Jo, S. M.; Park, S. K.; Sakong, J.; Sakong, J. Validity Assessment of Self-Reported Smoking Status in Firefighters Using the Urine Cotinine Test. Ann. Occup. Environ. Med. 2020, $32 \quad$ (1), $1-15$. https://doi.org/10.35371/aoem.2020.32.e2.

(7) Moyer, T. P.; Charlson, J. R.; Enger, R. J.; Dale, L. C.; Ebbert, J. O.; Schroeder, D. R.; Hurt, R. D. Simultaneous Analysis of Nicotine, Nicotine Metabolites, and Tobacco Alkaloids in Serum or Urine by Tandem Mass Spectrometry, with Clinically Relevant Metabolic Profiles. Clin. Chem. 2002, 48 (9),

https://doi.org/10.1093/clinchem/48.9.1460. $1460-1471$.

Gasperino, D.; Baughman, T.; Hsieh, H. V.; Bell, D.; Weigl, B. H. Improving Lateral Flow Assay Performance Using Computational Modeling. Annu. Rev. Anal. Chem. 2018, 11 (March), 219-244. https://doi.org/10.1146/annurev-anchem-061417125737.

(9) Lorkiewicz, P.; Riggs, D. W.; Keith, R. J.; Conklin, D. J.; Xie, Z.; Sutaria, S.; Lynch, B.; Srivastava, S.; Bhatnagar, A. Comparison of Urinary Biomarkers of Exposure in Humans Using Electronic Cigarettes, Combustible Cigarettes, and Smokeless Tobacco. Nicotine Tob. Res. 2019, 21 (9), 1228-1238. https://doi.org/10.1093/ntr/nty089.

(10) Jacob, P.; St. Helen, G.; Yu, L.; Nardone, N.; Havel, C.; Cheung, P.; Benowitz, N. L. Biomarkers of Exposure for Dual Use of Electronic Cigarettes and Combustible Cigarettes: Nicotelline, NNAL, and Total Nicotine Equivalents. Nicotine Tob. Res. 2020, 22 (7), 1107-1113. https://doi.org/10.1093/ntr/ntz235.

(11) Benowitz, N. L.; Bernert, J. T.; Foulds, J.; Hecht, S. S.; Iii, P. J.; Jarvis, M. J.; Joseph, A.; Oncken, C.; Piper, M. E. Biochemical Verification of Tobacco Use and Abstinence: 2019 Update. 2021, 22 (7), 10861097.

https://doi.org/10.1093/ntr/ntz132.Biochemical.

(12) Armbruster, D. A.; Pry, T. Limit of Blank, Limit of Detection and Limit of Quantitation. Clin. Biochem. Rev. 2008, 29 Suppl 1 (August), S49-52. 
(13) Jacob, P.; Goniewicz, M. L.; Havel, C. M.; Schick, S. F.; Benowitz, N. L. Nicotelline: A Proposed Biomarker and Environmental Tracer for Particulate Matter Derived from Tobacco Smoke. Chem. Res. Toxicol. 2013, 26 (11), 1615-1631. https://doi.org/10.1021/tx400094y.

(14) M. Chang, C.; H. Edwards, S.; Arab, A.; Y. Del VallePinero, A.; Yang, L.; Hatsukami, D. K. Biomarkers of Tobacco Exposure: Summary of an FDA-Sponsored Public Workshop. 2017, 1-26. https://doi.org/10.1158/1055-9965.EPI-160675.Biomarkers.

(15) Achilihu, H.; Feng, J.; Wang, L.; Bernert, J. T. Tobacco Use Classification by Inexpensive Urinary Cotinine Immunoassay Test Strips. J. Anal. Toxicol. 2019, $43 \quad$ (2), 149-153. https://doi.org/10.1093/jat/bky075.

(16) Bernert, J. T.; Harmon, T. L.; Sosnoff, C. S.; McGuffey, J. E. Use of Continine Immunoassay Test Strips for Preclassifying Urine Samples from Smokers and Nonsmokers Prior to Analysis by LCMS-MS. J. Anal. Toxicol. 2005, 29 (8), 814-818. https://doi.org/10.1093/jat/29.8.814.

(17) Parker, D. R.; Lasater, T. M.; Windsor, R.; Wilkins, J.; Upegui, D. I.; Heimdal, J. The Accuracy of SelfReported Smoking Status Assessed by Cotinine Test Strips. Nicotine Tob. Res. Off. J. Soc. Res. Nicotine Tob. 2002, 4 (3), 305-309. https://doi.org/10.1080/14622200210142715.

(18) Cooke, F.; Bullen, C.; Whittaker, R.; McRobbie, H.; Chen, M.-H.; Walker, N. Diagnostic Accuracy of NicAlert Cotinine Test Strips in Saliva for Verifying Smoking Status. Nicotine Tob. Res. Off. J. Soc. Res. Nicotine Tob. 2008, 10 (4), 607-612. https://doi.org/10.1080/14622200801978680.

(19) Jacob, P.; Hatsukami, D.; Severson, H.; Hall, S.; Yu, L.; Benowitz, N. L. Anabasine and Anatabine as Biomarkers for Tobacco Use during Nicotine Replacement Therapy. Cancer Epidemiol. Biomarkers Prev. 2002, 11 (12), 1668-1673. 